\title{
1)Le visa Schengen : expression d'une stratégie de " police » à distance
}

\section{Elspeth Guild et Didier Bigo}

\section{OpenEdition}

1 Journals

\section{Édition électronique}

URL : http://journals.openedition.org/conflits/924

DOI : $10.4000 /$ conflits.924

ISSN : 1777-5345

Éditeur :

CCLS - Centre d'études sur les conflits lilberté et sécurité, L'Harmattan

\section{Édition imprimée}

Date de publication : 1 mars 2003

Pagination : 22-37

ISBN : 2-7475-4868-8

ISSN : 1157-996X

\section{Référence électronique}

Elspeth Guild et Didier Bigo, «1)Le visa Schengen : expression d'une stratégie de « police » à distance », Cultures \& Conflits [En ligne], 49 | printemps 2003, mis en ligne le 29 septembre 2003, consulté le 30 mars 2021. URL : http://journals.openedition.org/conflits/924 ; DOI : https://doi.org/ $10.4000 /$ conflits.924

Ce document a été généré automatiquement le 30 mars 2021.

Creative Commons License 


\title{
1)Le visa Schengen : expression d'une stratégie de " police » à distance
}

\author{
Elspeth Guild et Didier Bigo
}

1) Le visa Schengen : expression d'une stratégie de " police » à distance

1 Depuis plusieurs années, nous avons développé la thèse que la coopération policière européenne se faisait en réseaux et à distance. Comme on le signalait dans un article de $1995^{1}$ on peut décrire «les politiques gouvernementales des pays européens en trois grands volets : le premier vise à bloquer ou dissuader les individus de partir de chez eux et c'est sans doute en ce domaine que les Européens, rattrapant les Américains, ont le plus innové depuis le sommet de Madrid de 1993 consacré aux visas et à la collaboration consulaire, car il s'agit de faire dela police à distance dans les pays d'origine et non d'attendre que les flux arrivent sur place. C'est ce que les euphémismes administratifs appellent une politique de 'prévention' ou 'proactive'. Le second volet vise à filtrer les demandeurs d'asile à la frontière en essayant de dégager le bon grain de l' ivraie, les bons réfugiés (c'est-à-dire ceux qui répondent de manière restrictive à l'application de la convention de Genève) de tous les autres que l'on dénommera faux réfugiés, réfugiés économiques, déboutés du droit d' asile. Le troisième volet vise à refouler, lorsqu'ils y sont déjà, hors du pays et hors d'Europe les candidats malheureux à l'asile. Tout ceci conduit à une nouvelle stratégie globale où la frontière conçue comme une ligne de partage entre deux Etats souverains disparait au profit d'une conception en termes de front, comme zone de souveraineté partagée, où les contrôles ne sont plus forcément réalisés par des autorités nationales mais par des autorités étrangères ou des compagnies privées, et une conception où ces contrôles touchent de moins en moins tout le monde pour s'appesantir uniquement sur certaines minorités. Ces logiques de contrôle et de surveillance n'ont plus de lieu fixe, de médiation fortement territorialisée et symboliquement attachée au ' bornage' territorial étatique malgré les discours de l'Europe forteresse ou de l'Europe passoire. Au contraire, elles se délocalisent en tout point du territoire national et au-delà pour la surveillance, elles s'articulent sur une médiation de l'apparence physique et cherchent symboliquement à se légitimer comme un nouveau 'bornage' d'une identité sociétale définie implicitement à travers la race, le civilisationnel, le culturel ». Et un peu plus loin il ajoutait: « Avec la politique de la liste commune des pays 
soumis à obligation de visa qui de 70 dans les années 85, 110 en 1990, est passée maintenant à 126 entre les pays Schengen (et atteint presque le même nombre dans les documents préparatoires à la signature à 12 de la convention sur les frontière extérieures), il a toutes les chances de devoir aller demander aux autorités consulaires d'un des pays signataires, son visa (avec le risque de se faire repérer par la police du pays qui a bien compris l'enjeu: (f. Togo). Or, la coopération consulaire entre pays Schengen sur place, qui est un des nouveaux développements de Schengen après le sommet de Madrid en Juillet 1993, semble plus soucieuse de lutter contre l'immigration illégale en refusant les visas de tourisme et autres que d'accorder des visas d'entrée à des demandeurs d'asile. Et dès lors que le refus de l'un vaut refus des autres, le choix initial devient crucial ». La réponse des autorités du ministère de l'Intérieur de l'époque à cet article qui résumait un rapport précédent était de refuser l'idée que Schengen était un instrument de politique migratoire et encore plus une ségrégation en rapport avec la pauvreté des demandeurs. Schengen était uniquement un moyen de lutte contre la criminalité et les illégalités, disait-on. La police ne s'occupe pas de l'asile ou de l'immigration. Les autorités consulaires n'ont qu'un rôle accessoire. Frontière et souveraineté sont liées. Le vrai contrôle est aux frontières extérieures.

Quand les ONG évoquaient le système "mondial visa " ou le système "vision ", de même que le rôle des officiers de liaison dans l' attribution des visas, la seule réponse était : exagération ; leur rôle est tout à fait marginal.

Dix ans après, en 2003, on se félicite ouvertement que les autorités consulaires soient responsables des contrôles préliminaires des frontières, qu'il y ait un tissu dense de relations entre les consulats permettant de filtrer les bona fide des mala fide et que la dissuasion à l'égard de ceux qui veulent venir sur le territoire Schengen soit efficace ${ }^{2}$. On admet que les contrôles aux frontières sont plus une surveillance générale qu'un contrôle individualisé et que, si contrôle il y a, il ne concerne que la conformité des documents et n'est nullement un contrôle des personnes. Mieux, on est fier de la collaboration consulaire et de la politique des visas. Celle-ci n'est plus honteuse. Pauvres touristes, demandeurs d'asile et migrants sont intégrés sans complexe et sans gêne au continuum de lutte contre la criminalité et le terrorisme.

Elspeth Guild a de manière détaillée (dans sa lecture inaugurale et dans des travaux précédents) montré les implications juridiques d'une telle politique ${ }^{3}$. Elle a insisté sur l'importance de cette transformation du sens de la frontière pour l'individu. Sa thèse permet de comprendre comment l'individu «active» de multiples contrôles et rencontre donc la frontière virtuellement avant de la passer physiquement. Selon que l'individu appartient à un pays membre de l'Union, à un pays tiers, à un pays soumis à obligation de visa, il ne rencontrera pas de frontière, ne la rencontrera qu 'une ou deux fois sur le territoire du pays d'arrivée ou la rencontrera dès le consulat et auprès des compagnies aériennes. Comme elle le signale, $\mathrm{l}^{\prime}$ individu active la frontière et les contrôles qui lui sont corrélés en amont dès qu'il décide de bouger ${ }^{4}$. Et elle l'explicite en montrant à quel point les contrôles varient selon l'individu, sa nationalité et son statut. La frontière se « déplace » et n'est plus liée au territoire sur lequel l'Etat a la prétention au monopole de la violence légitime. Elle devient celle d'autres Etats, les Etats Schengen à leur frontière extérieure, les consulats de ces mêmes Etats dans le pays de départ là où l'on exige des visas et pas simplement un passeport ${ }^{5}$.

\section{Police à distance, contrôle à distance et remote policing}

5 La notion de "police à distance » ou de « contrôle à distance » que nous développons tous les deux doit être précisée afin d 'éviter des confusions. Elle n'a pas de connotation 
organique et ne renvoie pas exclusivement aux pratiques des polices nationales. Elle désigne des pratiques de contrôle qui peuvent être réalisées par d'autres professionnels de la sécurité que les policiers comme par exemple les autorités consulaires. Elle ne vise pas exclusivement le report des contrôles aux frontières extérieures dans la perspective de l'élargissement, même si ce point peut en faire partie ${ }^{6}$. Des rapports parlementaires en France et aux Pays-Bas, tout comme certains écrits, ont repris cette notion pour évoquer le fait que les contrôles faits auparavant par la police nationale à la frontière se font maintenant par des polices étrangères sur la frontière extérieure de l' Union ou se font via des entreprises privées de sécurité engagées par des compagnies de transport à qui l'on délègue en partie la vérification des titres valides pour l'entrée aux frontières de l'espace Schengen?

6 La notion de police à distance a gagné en termes de précision et de contenu avec ces études mais, à une condition, c'est de ne pas réduire à ce seul point la notion en restant prisonnier de la logique du contrôle aux frontières de l'Etat Nation. Parler de police à distance c'est en comprendre la signification dans l'espace, au-delà de la frontière physique et même la signification dans le temps avec les logiques proactives tournées vers un futur possible.

7 A contrario d'un raisonnement focalisé sur la frontière physique étatique, il faut donc comprendre que la notion de frontière se détache du territoire au sens où elle n'en est plus la borne, la limite, l'enveloppe. La frontière a ainsi de plus en plus une signification différente où elle signale que, virtuellement, on passe de chez soi à chez l'autre. Elle est alors activée en termes de surveillance et de contrôle différemment selon les individus en jeu, selon leur "profil de risque ", car elle vise moins à protéger la souveraineté qu'à délimiter des identités en discriminant des «indésirables ». Le glissement du contrôle de la souveraineté territoriale au contrôle des identités nationales et sociales ainsi qu'au flux de personnes est alors ce qui permet de comprendre le sens des mesures à l'oeuvre. Certains étrangers ne voient quasiment plus de frontières car on les considère facilement comme des autres nous-mêmes - citoyens des autres pays de l'Union ou même de l'AELE et des Etats-Unis ou du Canada. En revanche, pour d'autres étrangers (extra-communautaires, appartenant à la liste noire, et pourvus de faibles moyens financiers), la frontière est activée à de nombreuses reprises ils ont beaucoup plus de chances d'être refusés à l'entrée ou, sans l'être forcément, d'avoir plus de désagréments administratifs et d'être dissuadés de se déplacer.

Plutôt que de parler de "police à distance ", on pourrait certes évoquer le " contrôle à distance " ou la "sécurisation à distance " afin de comprendre les logiques à l'ouvre, mais l'extension de la terminologie risque à l'inverse d'être sur-interprétée et d'être amalgamée avec des logiques militaires. Or, celles-ci sont différentes comme l'a montré Jean-Paul Hanon dans plusieurs de ses recherches ${ }^{8}$. Ce qui est en jeu avec la police à distance, c'est le contrôle de la circulation des personnes avant même leur entrée sur le territoire,mais ce n'est pas l' appréciation géopolitique de leurs effets et la mise en place de technologies de haut niveau visant un renseignement global.

En ce sens notre travail sur la police à distance ne recouvre pas tous les processus de sécurisation et d'insécurisation des logiques proactives, mais il est plus large que celui de remote policing pour signaler les envois de policiers, ou d'acteurs privés ayant des

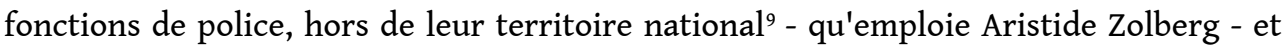
se rapproche des recherches capitales de Gérard Noiriel ${ }^{10}$ qui, sans utiliser le terme de police à distance, évoque les procédures d'identification à distance dans un sens très 
proche du nôtre. Ce sont ces procédures dont il a montré toute l' importance dans la constitution de l'espace national, à travers les ruptures de technologies de contrôle et de surveillance que cela a impliqué : passage des contrôle de face à face au sein de groupes à répertoire d'action locaux et patrimoniaux, à des contrôles de papier au sein de groupes à répertoire $\mathrm{d}$ 'action nationaux et de classe, qu'il faut suivre maintenant à l'échelle des frontières mouvantes de l'Union Européenne. Quelle est la relation entre le processus actuel d'européanisation et les technologies d'identification, de surveillance et de contrôle. Sommes-nous en continuité avec les pratiques des Etats nations ? Y a-t-il rupture avec la déterritorialisation de l' espace national des contrôles et sa reterritorialisation au sein de l'Union européenne, via les technologies de mise en réseau des bases informatiques de contrôle et de surveillance (SIS, Eurodac.) et ce dans une ère de globalisation de ces contrôles (EU-FBI, Echelon, espace du renseignement transatlantique en constitution.)? Peut-on parler d'un dispositif ban-optique mondialisé ?"11

Ces questions ont guidé notre réflexion et nous y répondrons de manière progressive tout au long de ce numéro en nous attachant à l'examen des pratiques des principaux acteurs concernés. Avant cela, il convient de considérer que la police à distance est une des composantes de la transformation des relations entre la guerre et le crime, entre l'action militaire et l'action policière, entre le civilo-militaire et la consolidation de la paix. Dans le volet des visas, la politique mise en place par les européens de Schengen vise à contrôler en amont pour éviter les querelles permanentes qui divisent les acteurs en ce qui concerne la prétendue abolition des contrôles aux frontières intérieures, le soi-disant renforcement des contrôles aux frontières extérieures et le transfert lié à l'élargissement des frontières extérieures à de nouveaux pays membres. Cette politique des visas a alors intérêt à se faire discrète, à rester à l'ombre des débats sur les frontières et à se parer des atours de la pure technique pour éviter des débats de fond sur les motivations de la mise à l'écart de certains étrangers.

11 Nous allons voir que les débats sur les frontières brouillent souvent la logique des visas, mais que la croyance dans la technologie d'identification des personnes via la biométrie pousse tous les acteurs à privilégier cette voie d'un contrôle sériant les " indésirables » sur le territoire européen et les individus à "risque migratoire ». Se pose bien sûr la question des relations entre les autorités gérant ces bases informatiques, l'identification de la personne et l'identification de ses papiers. Nous y reviendrons dans un prochain numéro sur les technologies de surveillance. Ici, nous n'allons développer qu'une petite partie de ce dispositif ban-optique dont les visas constituent un seul mécanisme, mais un mécanisme central dans la mise à distance, la mise à l'écart ${ }^{12}$.

L'attribution du visa et le rapport aux frontières : technologies d' identification et mise à l'écart

12 On retrouve au cour de la police à distance la question de l'attribution du visa. Et c'est la gestion de ce contrôle par des entités différentes des polices nationales aux frontières qui est en jeu. En effet c'est, au premier chef, le contrôle par les autorités consulaires dans les pays qui sont soumis à visa qui est important et non le contrôle aux frontières par les polices des frontières. Etant donné l'intensité des flux de personnes voyageant, le contrôle à la frontière est davantage une vérification des documents de voyage qu'un réel contrôle des personnes. Celui-ci a eu lieu avant, en amont lors de l'attribution de ces documents : passeports (et visas). Mais cela suppose que l'individu 
conserve avec lui ces documents et qu'il ne fraude pas. On verra que les peurs administratives reposent sur ce soupçon permanent et que cela explique pourquoi le passeport est de plus en plus dévalorisé au profit du visa dans la réflexion sécuritaire. Le visa, bien plus que le passeport, permet en effet un tri sélectif, de ceux qui pourront ou non circuler « librement». C'est une des limites de 1 'approche pourtant excellente de John Torpey concernant le rôle des Etats sur le territoire européen car il sous-estime fortement le rôle de l' élaboration de listes de pays soumis ou non à visa et néglige la dimension du rôle de la Commission Européenne comme acteur à part entière dans un processus qui se démarque du rôle de l'Etat comme garantissant ultimement le droit de circuler ${ }^{13}$. L'originalité de l'Union dans l'attribution de ce droit de circuler tient en effet à la combinaison entre passeport et visa et pas simplement à la délivrance des passeports. Et dans ce cadre la discussion sur les visas est plus significative que celle sur les passeports. La détermination de la liste des pays dont les ressortissants sont soumis ou non à visa marque en effet, qu'on le veuille ou non, une défiance à l'égard d'un pays ou d'une nationalité dans son ensemble. L'octroi du visa individuel est à cet égard une exception à l'exception (et c'est pourquoi elle obéit à une logique de "rareté » dans l'esprit de ceux qui les délivrent). C'est un rétablissement de la confiance à un individu après l'épreuve du soupçon porté sur un ensemble national. En cela, la situation $d$ 'accès au territoire et de circulation interne à l'Union diffère fondamentalement de celles des citoyens des pays qui n'ont besoin que d'un passeport car ici on continue de faire confiance à l'Etat d'origine pour dire qui est l'individu qui circule alors qu'avec les visas et $\mathrm{l}$ ' introduction de données biométriques au sein de ceux-ci on cherche à ne faire confiance ni à l'individu ni à son Etat mais à une « science » de l'identification.

Comme le signale la carte que nous avons établie, la liste des pays dont les ressortissants sont soumis à visa pour entrer dans l'Union Européenne/espace Schengen est une catégorie qui, en poussant la logique à l'extrême, a la forme euphémisée mais généralisée de la liste des « rogue states » à laquelle on aurait ajouter les pays à migration forcée. Elle signale une défiance, un soupçon, une peur. Défiance à l'égard de pays instables, de pays en conflits, de pays pauvres, de pays musulmans considérés comme radicaux dans leurs croyances. Elle instaure cette division du monde entre ceux qui vivent de, par et pour la circulation (les élites mondialisées) et ceux qui souffrent d'être piégés dans le local dans un monde qui valorise les images de mobilité, de fluidité, de vitesse ${ }^{14}$. Mais jusqu'où les pratiques de circulation sont-elles affectées par cette défiance? La question est des plus délicates. Les représentations du monde ont des effets sociaux et provoquent la souffrance la plus réelle, mais des contradictions pratiques, des résistances empêchent la cartographie voulue par certains acteurs de l'Union Européenne d'être mise en pratique.

Les enjeux de l'élargissement : le déplacement des contrôles aux frontières, confiance et défiance, aller au-delà des frontières

On comprend par exemple pourquoi un des premiers objectifs politiques des pays soumis à l'obligation de visa est d'être retiré de cette liste, surtout s'ils sont dans la liste des candidats à l'Union. Ils comprennent que leur chance d'entrer un jour dans l'Union est conditionnée par l'abandon de la défiance et l'acceptation de la règle du simple passeport pour leurs citoyens. C'est ici où l'exemple bulgare traité par Elena Jileva dans son article a tout son intérêt pour notre recherche. En effet, il apparaît à la lecture de sa recherche de terrain que le fait de retirer la Bulgarie de la liste des pays soumis à visa tient bien moins aux mesures effectives que celle-ci prend en matière d'harmonisation des listes de pays soumis à visa ou des problèmes dits de migration 
clandestine traversant son territoire en direction de l'Union que des engagements symboliques que les autorités bulgares sont prêtes à faire sur la corruption, sur la législation globale, sur l'influence économique de l'Union par rapport aux Etats-Unis. Et d' ailleurs lors de la crise irakienne, le président Jacques Chirac l'avait rappelé sèchement aux pays candidats. Les ministères des Affaires étrangères et le COREPER ainsi que la Commission dans les services liés au marché unique veulent avant tout créer une "dynamique positive » et veulent éviter la constitution de "zones tampons » ralentissant les activités économiques autour de l'Union européenne et détruisant les solidarités commerciales et culturelles qui existent " pour simplement faire plaisir à une certaine extrême droite sécuritaire $\aleph^{15}$. Pologne, Bulgarie et Roumanie, Ukraine doivent maintenir des liens économiques et culturels. Il n'est pas question de les détruire par l' imposition de contrôles frontaliers drastiques. Les Polonais et les Bulgares ont été rassurés sur ce point. On leur demande du symbolique et non du concret ${ }^{16}$.

Entre le discours officiel sur l'acquis Schengen, maintenant intégré à l' acquis de l'Union, que les candidats doivent théoriquement mettre en ouvre et leurs capacités d'appliquer ces mesures, personne n'est dupe ${ }^{17}$. On leur demande de «faire des gestes de bonne volonté » en matière de législation, en contrepartie de quoi on lèvera en partie les mesures de défiance et on financera des équipements ${ }^{18}$. En termes politiques, il n'est donc pas certain que la question des frontières et des visas que devraient mettre en place les pays candidats soit liée sur le fond aux impératifs de sécurité et de migration malgré la politique symbolique visant à en convaincre l'opinion et visant à donner des gages aux professionnels de la sécurité. Il s'agit de faire disparaitre la suspicion à leur égard, de les «normaliser », de les présenter comme "nous » et non plus comme " eux », et sans pour autant les obliger à déclarer les mêmes ennemis.

16 Ce n'est pas la stratégie des principaux acteurs JAI qui tiennent à cette bourse d'échange des inquiétudes comme ciment de l'Union. Ils insistent sur le rôle des contrôles aux frontières et sur les visas. Les impératifs de sécurité sont évoqués avant tout par les autorités des pays qui ne veulent pas d'un élargissement rapide aux candidats de la seconde vague et plaident pour une "pause». Pour eux, la libre circulation des personnes des pays candidats est dangereuse et doit de plus en plus être mise en question y compris pour les pays de la première vague. En effet, on donne les exemples grecs et autrichiens pour rappeler que le principe d'entrée dans la convention Schengen n'a pas été suivi d'effets immédiats et qu'il leur a fallu prouver d'abord à un comité composé des pays membres, l' effectivité de leur capacité de contrôle pour qu'on accepte la libre circulation. Fort de ce " précédent », les contrôles aux frontières germano-polonaises ne seraient donc pas levés aussi rapidement qu 'on l'a dit et ce, même si l'on demande à la Pologne d'instaurer des contrôles renforcés à sa frontière orientale ${ }^{19}$. Le ministère de l'Intérieur allemand évoquait en juin 2001 une période de transition de sept ans mais un fonctionnaire du BKA lors du colloque CEPS (6 et 7 juillet 2001) plaidait pour dix ans et ce, à partir de l'entrée effective dans l'Union des pays candidats. Certains autres pays, dont la France depuis peu, ont aussi ce genre de discours voulant mettre au pied du mur les pays candidats et les obliger à casser leurs liens traditionnels économiques et culturels.

Il n'y a là aucun "paradoxe libéral » lié à la situation migratoire comme on l'évoque parfois, mais plutôt contradiction entre des réseaux bureaucratiques transnationaux soutenant des intérêts privés différents ${ }^{20}$. Les contrôles sont déniés par ceux qui veulent que, politiquement, l'Union soit le plus rapidement élargie afin de ne laisser 
personne en dehors, avec les risques de frustration que cela ferait naître. Ceux qui croient aux arguments sécuritaires proviennent avant tout des ministères de la Justice et de l'Intérieur alors que les ministères des Affaires étrangères ou les ministères du Travail valorisent beaucoup plus l'élargissement en termes $d$ ' apport positif - dans la concurrence avec les Etats-Unis et/ou en termes de main d'ouvre qualifiée.

Si le raisonnement, majoritaire au conseil JAI, mais combattu par la Commission et le Parlement, est suivi, on aura donc un découplage entre l' espace de libre circulation qui ne serait assuré qu'entre certains pays - les plus anciens dans la collaboration et les plus riches - et l'espace de collaboration policière qui, lui, existerait entre tous les pays ayant accepté l'acquis Schengen. Ce découplage semble d'ailleurs avoir été compris par le Royaume-Uni qui joue sur celui-ci pour maintenir les contrôles à ses frontières mais qui participe à toute la collaboration concernant le contrôle à distance. Dès lors, pour la Hongrie ou la Pologne, comme pour la Roumanie et la Bulgarie, l'enjeu d'une future libre circulation serait bien plus éloigné qu'ils ne l'imaginent avec les dates symboliques de 2004 et de 2007. Peut-on parler d'un jeu de dupes entre l'Union et les pays candidats? Jusqu'où est-on prêt à les accepter pleinement et à revoir de fond en comble notre conception de qui est l'Autre, de qui est l'Européen, le Communautaire?

Les décisions importantes prises par le Conseil Européen à Copenhague en décembre 2002 qui portaient sur la libre circulation des personnes venant des dix pays candidats dès le 1er mai 2004 et 2007 pour la vague suivante ont suscité des réactions en Allemagne et en France. Les professionnels de la sécurité et une partie de la droite y ont vu un laxisme coupable. Inversement les Pays-Bas, la Suède, l'Espagne, l'Irlande et le Royaume-Uni ont déclaré qu'il n'y aurait pas de période transitoire. Quand on sait que les Britanniques ont maintenu les visas pour les Roumains et les Bulgares, qu'ils ont menacé les Tchèques de les réinstaurer en profitant du fait qu' ils ne sont pas liés par les décisions de Schengen sur la libre circulation, comment expliquer que le RoyaumeUni ait pris la tête, une fois n'est pas coutume, de la campagne en faveur d'une libre circulation immédiate des pays candidats $?^{21}$ Une des explications tient peut-être à la concomitance d' événements différents. En effet, les pays candidats à l'Union se sont rappelés cette position récente lorsqu'il leur a fallu se positionner pour ou contre l'intervention en Irak et leur alignement sur la position britannique (plus qu'américaine) contre la position franco-allemande est peut-être lié en partie à la plus grande ouverture proposée par certains pays $^{22}$. La réunion symbolique à l'Acropole d'Athènes a entériné une fois de plus le principe de frontières ouvertes, y compris à l'extérieur, la création d'un cercle d'amis et le refus d'une Europe frileuse repliée derrière des frontières forteresses ${ }^{23}$. Les combats se déplacent donc encore plus sur une ligne de contrôles moins visibles que ceux aux frontières extérieures, les contrôles concernant ceux qui peuvent voyager dans l'Union, et sur la quantité de visas que l'on peut et que l'on doit attribuer. La question des listes blanches et noires de pays, d'imposition ou non de visas semble alors plus nous renseigner sur les stratégies effectives de contrôle que la question des contrôles aux frontières. Elle en dit plus sur les impératifs de sécurité et de migration ; sur la construction sociale de peurs plus ou moins partagées concernant l'Autre; ainsi que sur la manière dont les Européens cherchent à se donner une image d'eux-mêmes, une identité commune. La question de l'attribution individuelle de visa renvoyant à une invisibilisation et une délocalisation en amont des contrôles visant à en masquer les catégories d'attribution et le caractère aléatoire qui tourne à la loterie. 
La logique juridique du visa Schengen : la réallocation des identités souhaités par l'un des Etats participant ne soient pas autorisés à entrer sur le territoire. Ainsi, les règles se concentrent sur qui doit être exclu et fournissent très peu d'indications sur qui peut être admis. En raison du principe sous-jacent du système, à savoir la reconnaissance croisée des décisions nationales plutôt qu'une harmonisation, trouver des moyens légaux à leur mise en place a des implications inattendues. La suppression des contrôles aux frontières entre les Etats signifie, par exemple, que les décisions positives d'admission d'individus sont probablement respectées par défaut ainsi les «Parties Contractantes ${ }^{24}$ ont à diligenter moins de contrôles d'identité aux frontières mais leur méfiance à l'égard des autres administrations peut les pousser à les remplacer par des contrôles renforcés à l'intérieur du pays, dans certaines zones quartiers dits difficiles - ou à l'égard des lieux de vie des minorités étrangères extra communautaires ${ }^{25}$. La reconnaissance croisée des décisions négatives requiert, elle, des mesures encore plus spécifiques. Quand le concept de sécurité intérieure (la raison première du refus d'admission d'un individu au sein du territoire Schengen) n'est pas harmonisé, tout examen des raisons d'un refus d'un individu par un autre Etat permet d'être évité. Aux Pays-Bas, le mécanisme juridique pour réaliser cela se trouve à l'Article 109 (4) et (5) de la loi sur les étrangers de 1999, qui situe la frontière néerlandaise pour la circulation des personnes aux extrémités de tous les Etats Membres et incorpore la sécurité intérieure de tous les Etats Membres à la sécurité intérieure néerlandaise. Mais une telle conséquence est refusée par d'autres Etats qui tiennent à mettre en avant une rhétorique souverainiste. Dès lors, malgré l'hétérogénéité des applications locales dont nous verrons les incohérences de fond, on peut résumer la logique juridique déclarative du visa Schengen en dégageant trois principes.

Premier principe: aucun ressortissant d'un Etat tiers ne peut obtenir l' accès au territoire des Etats Schengen (avec ou sans visa de court séjour) s 'il est susceptible de constituer un risque à la sécurité de l'un des Etats membres. Il faut donc pour chaque Etat connaitre les individus considérés comme des risques à la sécurité des autres pays, en même temps qu'à la leur.

Cela se fait via la consultation des bases de données de police nationales, des bases internationales comme celles d'Interpol et via le lien entre partie des bases de données nationales des autres pays de l'Union créé par le fonctionnement du SIS et du SIRENE ((Supplément d'Information Requis à l' Entrée NationalE / Supplementary Information Request at the National Entry). L'on trouve au sein du SIS, les noms des individus qui ont déjà été sur le territoire de l'Union et qui, pour un motif ou un autre y sont depuis «indésirables». Cela se traduit pour eux par l'inscription sur la liste de ceux ne pouvant plus être admis. Cette liste - résultat de l'attitude à tenir des autorités à l'entrée du territoire ou lors de la délivrance des visas - est maintenue électroniquement au sein du SIS, et elle est composée de toutes les personnes signalées 
par les Etats participants en fonction de leur compréhension nationale du critère d'inclusion et de leur propre interprétation de l'ordre public et de la sécurité (article 96 de la Convention d'Application de l'Accord de Schengen de 1990). La définition de ces personnes comme «indésirables » est fondée sur ce qu'ils ont fait ou ont représenté quand ils étaient sur le territoire de l'Union. C'est ici que la question des conceptions divergentes de ce qui constitue un risque et de ce qu'est la sécurité devient centrale. Ce qui est perçu comme un risque à la sécurité dans un Etat n'est pas forcément identique dans un autre. Cette différence dans la perception du risque, comme elle se réfère aux activités de l'individu la dernière fois qu'il se trouvait dans l'Union, se fera soit lors de l'attribution du visa, soit plus tard dans le territoire où les tribunaux commenceront à remettre en question la légitimité du système. La discussion sur la pertinence de l'inscription au SIS et les variations nationales ne fait que commencer. Néanmoins, dans le cas des personnes inscrites au SIS, on peut considérer que sauf erreur d' homonymies, la relation entre individu et risque est faite sur la base d'un individu déjà connu et ayant déjà séjourné sur le territoire de l'Union. Ce n'est pas le cas en revanche pour ceux qui déposent pour la première fois une demande de visa et se le voient refuser $^{26}$.

Deuxième principe, il existe de la part des Etats une présupposition de légalité concernant un visa de court séjour délivré par l'un des autres Etats participants ${ }^{27}$. Dès lors, il sera reconnu comme valide pour pouvoir entrer sur le territoire commun (même s'il y a quelques exceptions explicites qui peuvent justifier le refus, expressément pour des raisons de sécurité et qui relèvent des polices aux frontières). Cela suppose qu'il existe un système de coordination et de délivrance des visas suffisamment légitime et précis pour que chacun accepte les documents des autres autorités et ne surajoute pas son contrôle au contrôle précédent. Cela suppose aussi une confiance réciproque marquée par la croyance dans la vérité des contrôles effectués par les administrations des autres pays membres. Théoriquement, le visa « uniforme " Schengen est fait pour éviter ce désagrément pour les personnes de multiples contrôles et comme gage de la confiance réciproque. Néanmoins, en pratique, des dysfonctionnements existent. Pendant longtemps, ils perduraient à deux niveaux, celui de la liste des pays soumis à visa et celui des individus auxquels il fallait refuser un visa. La Commission Européenne a réussi à faire éliminer, via le conseil JAI, depuis décembre 1998, les divergences entre les Etats sur la liste des pays à risques ${ }^{28}$. Il n'y a plus de liste grise même si le cas de certaines nationalités ou des réfugiés est toujours posé. En revanche, les critères d' attribution des visas pour pouvoir entrer sur le territoire commun pour de courts séjours (visites inférieures à trois mois) ne sont pas interprétés de la même manière par les agents consulaires des différents Etats ${ }^{29}$.

Troisième principe, une fois sur le territoire commun, l'individu est autorisé (mais cela peut aussi être sujet à exceptions pour des raisons de sécurité) à se déplacer sur tout le territoire pendant une période de trois mois sans aucun contrôle supplémentaire aux frontières intérieures des Etats participants. Ce principe de libre circulation interne est censé compenser les gênes occasionnées dans les phases précédentes. Mais, en pratique, les contrôles d'identité sont activés pour tous ceux qui dérogent à l'image que l'on se fait de l'Européen ou de l'étranger bienvenu. Le critère de la pauvreté est alors avec celui de la couleur de la peau l'un des plus puissants déclencheurs de contrôle ${ }^{30}$. 


\section{NOTES}

1. . Didier Bigo, «Les Etats face aux flux transfrontières de personnes : enjeux et perspectives ", Cahiers de la sécurité intérieure, n 19, 1995.

2. . Communication de la Commission au Conseil et au parlement européen concernant une politique commune en matière d'immigration clandestine. Com 2001 (672) Final, en particulier mesures en amont des frontières (Cf. Cultures \& Conflits, ${ }^{\circ} 50$, Eté 2003).

3. . Elspeth Guild, Moving the Borders of Europe, University of Nijmegen, 2001 ; Elspeth Guild «Intersecting Worlds? The European Community and the International Framework of Labour Migration », International Studies Association Conference, April 2000, Los Angeles.

4. . « One important physical manifestation of borders results from attempts by individuals to move. The individual, through interaction with state and other actors over the granting or withholding of rights, activates the 'border' and engages with the government regarding the position of the border ». Moving the borders of Europe, lecture inaugurale, op. cit.

5. . « For example, a Polish national driving in her car to Berlin will encounter the EU border for the first time at the physical edge of Germany. A US national arriving at Schipol airport directly by plane from New York will encounter the EU border first at check-in in New York when his passport is examined by the airline staff and security officers there for the purpose of controlling the EU border. He will then re-encounter the EU border when he must pass through immigration control at Schipol airport. A Moroccan national first encounters the EU border at the French consulate in Rabat when she seeks a visa. She will then re-encounter the border when she seeks to check in to catch her flight to Paris. She will again find the border when she arrives at Roissy Charles de Gaulle airport and passes through immigration control. So it is the individual who finds the border by virtue of his or her intentions and action relating to movement. But what is the border he or she activates? Dutch law provides at Article 109(4) Aliens Act 2000 that the borders of the Netherlands for the admission of aliens is to be found at the edge of the frontiers of all the Schengen states. Thus Germany, France, Italy etc are part of Dutch sovereignty for the purpose of the borders for persons. Further, Article 109(5) goes on to provide that "national security" of the Netherlands for these purposes means the national security of all the Schengen states. Returning then to the Weberian definition of the state, the enforcement of order over a defined territory no longer applies to the Member States as regards movement of persons. Access to the territory is controlled by a network of bureaucracies acting in accordance with the principle of cross recognition of their decisions » in her inaugural lecture Moving the Borders of Europe which was delivered during the official ceremony on the occasion of the assumption of the professorship of the CPO Wisselleerstoel at the University of Nijmegen, the Stichting Steunfonds Juridisch (Post) Doctoraal Onderwijs on 30 May 2001, and published by University of Nijmegen.

6. . Bigo Didier, «Border Regimes and Security in an Enlarged European Community : Police Co-operation with CEECs : between trust and obligation », in Jan Zielonka, Europe unbound : enlarging and reshaping the boundaries of the European Union, Routledge, 2002, pp. 213-240. 
7. . Masson Paul, Rapport sur la convention d'application de l'accord de Schengen, 31/01/96 ; "Schengen », in Revue droit et défense 2/1996. Hanon Jean-Paul, La fonction de protection, in Bigo Didier, Bonelli Laurent, Tsoukala Anastassia, Rapport pour le Centre de prospective de la gendarmerie, 2000 ; Guiraudon Virginie, « Logiques et pratiques de l' Etat délégateur : les compagnies de transport dans le contrôle migratoire à distance ", Cultures \& Conflits, De Tampere à Séville (1), n5, 2002, pp. 51-79. Guiraudon Virginie "Before the EU Border : Remote Control of the "Huddled Masses" » in Guild Elspeth, Minderhoud Paul, Borders of the European Union, Kluwer Law International, 2003.

8. . Hanon Jean-Paul, « La fonction de protection », op.cit.; Hanon Jean-Paul, « Les coopérations policières aux frontières Schengen germano-tchèque et germanopolonaise ", Cahiers de la sécurité intérieure, Polices post communistes, 41, 3/2000, pp. 175-191.

9. . Zolberg Aristide, « Commentary on Current Refugee Issues », Journal of International Affairs, 1994, 47 (2), 341-350 ; Zolberg Aristide et al., Escaping from Violence : Conflict and Refugee Crisis in the Developing World, New York, Oxford University Press, 1989 ; Zolberg Aristide, « International Migration Policies in a Changing World System », in Mcneill W., R. Adams (ed), Human Migration, Bloomington, Indiana University Press, 1978, pp. 241-286.

10. . Noiriel Gérard, Etat, nation et immigration vers une histoire du pouvoir, Belin, Paris, 2001.

11. . Bigo Didier, "The globalisation of (in)security », London, Beijin, Traces, 03/2003. Nous y expliquons que le ban-optique ne repose plus sur l'immobilisation des corps sous le regard analytique du surveillant mais sur l'extraction de la différence signifiante, sur l'exceptionalisation par rapport à la norme et sur la rapidité avec laquelle on « évacue ». Le dispositif de la nouvelle surveillance prend une autre forme qui rappelle les technologies de l' informatique et de la réalité virtuelle. Ce dispositif apparait comme le montage virtuel (morphing) de toutes les positions des individus au sein des flux. D'une image initiale (l'immigré, le jeune de banlieue) à une image finale (le terroriste, le trafiquant), on reconstitue virtuellement toutes les étapes de la transformation.

12. . Sur le dispositif ban-optique voir « The globalisation of (in)security » in Traces, op.cit.

13. . Torpey John, The Invention of the Passport, Surveillance, Citizenship and the State, London, Cambridge University Press, 2000, 211 p.

14. . Bauman Zygmunt, op.cit.

15. . Entretiens réalisés par Didier Bigo avec plusieurs hauts fonctionnaires, membres de la Commission ou détachés par les autorités nationales (avril 2002).

16. . Néanmoins, d'après Guy Pierre Chomette, le prix des visas décourage Ukrainiens et Biélorusses d'aller en Bulgarie et de profiter de la mer noire. Les flux touristiques se réorientent vers la Crimée. « Sur les marches orientales de l'Union Européenne » in Le Monde Diplomatique, mars 2003, pp. 6 et 7.

17. . Voir Amato Giuliano et Bart Judith, Final Report of the reflection Group on « The long -term Implications of EU Enlargement : the nature of the new Border », Badia Fiesolana/ Italie, European University Institute, novembre 1999. Voir également Bigo Didier, «La coopération policière avec les PECO : entre confiance et exigence », Les Cahiers de la Sécurité Intérieure (41), Paris, IHESI, 2000. 
18. . Voir Savona Ernesto U., « Dynamics of Migration and Crime in Europe : New Patterns of an old Nexus », Courmayeur, ISPA C/Conférence de (octobre 1996). 19. . Ici nous avons des informations contradictoires. Les déclarations politiques en faveur de la fin des contrôles à la frontière orientale de l'Allemagne se multiplient mais les fonctionnaires parlent du maintien et du renforcement des contrôles polonais à leur frontière occidentale.

20. . Hollifield James, Immigrants, Markets, and States : The Political Economy of Postwar Europe, Cambridge, Harvard University Press, 1992 ; Torpey John, The Invention of the Passport. Surveillance, Citizenship and the State, Cambridge, Cambridge University Press, 2000 ; ou Virginie Guiraudon (in Guild Elspeth, et Minderhoud Paul, Borders of the European Union, Kluwer Law International, 2003) évoquant un triangle entre normes libérales, politiques populistes et économie globale. A contrario de cette croyance plus ou moins forte dans le paradoxe : Guild Elspeth « Intersecting Worlds ? Understanding the Legal Framework of European Labour Migration in Susan Strange's Modern World », IPSA Québec 2000, ainsi que le panel de l'ISA, « The liberal paradox into question » présenté par Elspeth Guild et Andrew Geddes, Nouvelle Orléans, 2002.

21. . Nous reviendrons dans les pages suivantes sur la position singulière du RoyaumeUni et sur la diversité des positions des Etats membres.

22. . D'après nos entretiens en France, le Royaume-Uni a considéré que cela affaiblissait le discours des pays Schengen d'être à la pointe du progrès en matière de libre circulation en les prenant de vitesse et que cela lui permettrait d'être le pays attirant immédiatement la main d'ouvre hautement qualifiée. Stratégie voulue surtout par Barbara Roche ancienne responsable au ministère de l'Intérieur de cette politique visant à combler les déficit en main d'ouvre des secteurs de la santé et de l'informatique. Mais qui a dû démissionner.

23. Sur la notion de cercle d'ami voir la communication de la Commission sur l'élargissement de 1 'Europe Com (2003) 104-final.

24. . La terminologie « Parties Contractantes » est celle utilisée à l'intérieur de la Convention d'application de Schengen. Les rédacteurs ne pouvaient dire « les pays membres de l'Union » et ont donc utilisé ce terme.

25. . Voir l'article de Keith Groenendijk sur « Le maintien des contrôles des personnes aux frontières intérieures après l'entrée en vigueur de l'Accord d'Application Schengen en 1990 : Article 62 CE et Frontières de l'UE » in Guild E., Minderhoud P., In search of Europe's borders, Kluwer Law International, The Hague, 2003.

26. . Guild E., Moving the borders of Europe, op. cit.

27. . La présupposition en droit est inférieure à l'idée d' une présomption.

28. . Voir l'article de Gérard Beaudu, op. cit.

29. . La recommandation du Conseil relative à la coopération consulaire locale en matière de visas (troisième pilier) incite à « la coopération locale sur les visas, comprenant un échange d'information sur les critères de délivrance des visas et un échange d'information sur les risques pour la sécurité nationale et l'ordre public ou sur les risques d'immigration clandestine " (Article 1 JO 1996 C 80/1). Les contrôles sur la propriété de l'information ne sont pas inclus bien que la Recommandation poursuive « leurs services consulaires devraient échanger l'information afin d'aider à l'évaluation de la bonne foi et de la réputation des demandeurs de visa, ceci signifiant que le fait que le demandeur de visa en ait obtenu un pour un autre État Membre n'empêche pas les autorités d' examiner individuellement la demande de visa et de procéder aux 
vérifications requises pour des raisons de sécurité, d'ordre public et de contrôle d'immigration clandestine » (Article 6). Les concepts d'ordre public et de contrôle de l'immigration clandestine ne sont pas définis.

30. . La suspicion continue souvent à l' intérieur des frontières des pays de l'Union et touche par exemple les jeunes issus de l'immigration, même s'ils sont citoyens du pays en question. Nous ne développerons pas ici les technologies des contrôles d'identité à l' intérieur des frontières et aux frontières intérieures, mais il n'est pas possible d'en faire entièrement l'économie. Le moment de la police à distance ou en amont ne prend son sens qu'en relation avec le moment de la police en aval, à l'intérieur de l'espace. Voir Bigo Didier, « La mondialisation de l'(in)sécurité » in Traces, op. cit.

INDEX

Mots-clés : construction européenne, police à distance, Visa

Index géographique : Schengen

Thèmes : Schengen (convention) 